\title{
L'atopie ou le processus de désencombrement
}

Une lecture de The Blue Road de Kenneth White

\section{Christophe Roncato}

\section{(2) OpenEdition}

Journals

Édition électronique

URL : https://journals.openedition.org/etudesecossaises/73

DOI : $10.4000 /$ etudesecossaises.73

ISSN : 1969-6337

Éditeur

UGA Éditions/Université Grenoble Alpes

Édition imprimée

Date de publication : 30 janvier 2008

Pagination : 79-90

ISBN : 978-2-84310-110-6

ISSN : 1240-1439

Référence électronique

Christophe Roncato, "L'atopie ou le processus de désencombrement », Études écossaises [En ligne], 11 | 2008, mis en ligne le 30 janvier 2009, consulté le 21 septembre 2021. URL : http://

journals.openedition.org/etudesecossaises/73 ; DOI : https://doi.org/10.4000/etudesecossaises.73

Ce document a été généré automatiquement le 21 septembre 2021.

(c) Études écossaises 


\title{
L'atopie ou le processus de désencombrement
}

\author{
Une lecture de The Blue Road de Kenneth White
}

\section{Christophe Roncato}

My innermost being leaves in a country that does
not exist, but is more alive than all the nations,
all the institutions, all the constituted forms.
Kenneth wHITE, Pilgrim of the Void.

1 Une des caractéristiques majeures des travaux de création, tel qu'un poète comme Kenneth White les conçoit, consiste à résister à l'atrophie intellectuelle provoquée par le monde moderne. La sensation d'enfermement que cet enveloppement engendre est en étroite corrélation avec la question du lieu et la façon qu'a l'homme moderne de l'appréhender. Les lieux, ou topos, ont une place privilégiée dans l'œuvre de l'auteur franco-écossais : lieux de résidence (Paris, l'Ardèche, Pau, Trébeurden) et de " passage " (l'Europe, le Sud-Est asiatique, l'Amérique du Nord), sont systématiquement au centre de ses préoccupations. Au fil des séjours et des pérégrinations il devient clair que le lieu n'est par pour Kenneth White un moyen de se laisser aller à des fantasmes et, via l'imagination, d'échapper à la réalité, mais plutôt, d'éprouver pleinement le réel. L'ailleurs imaginé, signe distinctif de l'utopie, n'est pas moteur de la pensée ni de l'écriture. Au contraire, c'est l'ici vécu qui donne l'impulsion. White cherche à densifier sa sensation du monde au contact du lieu qu'il explore. C'est, selon lui, en repensant son rapport au topos, en essayant de l'occuper et de l'habiter, que l'individu peut en jouir pleinement. Cette « conception » du lieu, espace ouvert par excellence, est moins spécifique, que générique, moins cloisonnée que rhizomique

But what's a blue road... I'm not too sure about that myself. There's the blue of the big sky... the blue of the river, the mighty

St. Lawrence... the blue of the ice... Maybe the blue road is that passage North, among the blues of silent Labrador. (p. 11, 12)

La route bleue est un espace composite dont la définition se donne difficilement. Mais est-ce réellement un lieu, une portion déterminée de l'espace ? Kenneth White cherchet-il à repenser si radicalement l'approche du lieu qu'il en devient impossible d'en 
donner une définition? L'hétérogénéité de cette route bleue la place hors cadre, en marge. Elle est paradoxalement atopique, en route vers une nouvelle conception du lieu.

The Blue Road relate les pérégrinations de l'auteur sur la côte est du Canada. Le récit commence à Montréal et se termine au bord de la baie, septentrionale, d'Ungava. Il explore avec minutie et délectation le territoire parcouru en mettant l'accent sur le contraste entre la nature (espace ouvert) et le monde "civilisé » (espace clôt et clôturant). Hormis quelques incursions dans les terres, la route suivie longe le littoral :

The Labrador coast, writes a geologist, is still one of the most bold and rugged in

the world... Mother Nature has here taken off more than the usual amount of clothing... (p. 127)

Espaces vierges, côtes découpées et climat difficile déterminent les lieux. Le cheminement, qui mène le lecteur du sud vers le nord et d'un espace urbain vers un espace désertique, sert de colonne vertébrale au récit et reflète le désir d'épure cher à l'auteur. La structure du texte en témoigne : les chapitres sont courts, l'écriture dense et synthétique, le récit se termine par un poème. Ce travail de désencombrement du texte fait écho aux questions ontologiques de l'auteur :

For years I've been trying to get out from under a big book and all the mind-fuzz it engendered. I've been trying to make my way out of the Jehovian occupation of the world. All clear now. But there remains the final step. That's Labrador. Labrador is where I come full circle, swa-llow my birth, develop all the negatives of my adolescence and get a good look at my original face. (p. 21)

La métaphore du cercle est au cœur de l'ouvrage. De la première étape, Montréal, à la dernière, Ungava («the farthest place »), s'effectue une révolution qui donne naissance à un être revitalisé. Une double dynamique s'instaure au sein de ce parcours circulaire, avec d'un côté un processus de déconstruction et de l'autre une phase de (re-)construction. Kenneth White est un homme de la post-modernité soucieux de repenser le monde dans lequel il vit. Néanmoins le changement culturel qu'il envisage n'est pas centré sur un projet sociétaire. S'il reste admiratif d'un homme comme Fourier ${ }^{1}$ (cf. Un dernier regard sur l'Utopie, dans Une stratégie paradoxale, 1997), il reconnaît en revanche les limites de la pensée utopiste, si diverse soit-elle, et fait passer la question de l'être et du développement de l'esprit au premier plan. L'« atopos » est le lieu où l'être peut se mouvoir librement et augmenter sa sensation de vie. L'atopie est alors le site vierge où l'être peut exister, c'est-à-dire sortir de lui-même et se réaliser.

Quelles possibilités a l'homme moderne pour se désenclaver, pour sortir du marasme et de l'étourdissement dans lesquels il est plongé? L'épigraphe mise en tête du récit donne une première réponse : "For all minds tempted by the Outside, ready to turn nomad». Le nomadisme, terme clef de la pensée whitienne (cf. L’Esprit nomade, 1987), constitue la base d'une pensée du mouvement, d'une pensée qui ne se fixe pas de manière définitive en un lieu pré-établi mais qui pourtant en tire une réelle jouissance. Cette nouvelle disposition de l'être le met en contact direct avec l'extérieur. Le travail de désenclavement se met en place à deux niveaux : la sortie du monde moderne doit se faire physiquement (s'écarter de la foule) et mentalement (repenser les fondements de l'être). La dichotomie (intérieur/extérieur) est un trait caractéristique de The Blue Road. White met en tension des couples de nature opposée: ville/nature, pensée de l'intériorité/pensée de l'extériorité. Ce mouvement de diastole et de systole signale un parcours, un processus de désencombrement que nous suivrons dans son évolution. 
7 Tous les récits de voyage de Kenneth White commencent dans une ville: Travels in the Drifting Dawn (1978) à Londres, Pilgrim of the Void (1980) à Hong Kong, The Blue Road (1983) à Montréal... et s'achèvent dans des lieux sauvages. La structure des récits indique clairement une distanciation vis-à-vis du milieu urbain, le passage d'un lieu encombré à un espace ouvert. L'univers de la ville est largement représenté dans The Blue Road: les exploitations minières, les logements précaires et autres lieux de divertissement sont mentionnés jusqu'aux abords de la baie d'Ungava, avant-dernière étape du voyage. Il y a donc un va-et-vient incessant entre ce que Deleuze et Guattari appellent les espaces « lisses » et les espaces « striés $^{2}$ ». « L'espace lisse et l'espace strié, - l'espace nomade et l'espace sédentaire, - l'espace où se développe la machine de guerre et l'espace institué par l'appareil d'État, ne sont pas de même nature » (Mille Plateaux, p. 592). L'espace lisse, par nature hétérogène et non " pré-déterminé », laisse une grande liberté à l'individu ; à l'inverse l'espace strié, via une organisation stricte, fonctionne comme un organe régulateur.

Fort Chimo... is a huddle of red and green shacks... It's civilized, all right. You can play billiards, you can play Bingo. You can go to the cinema run by the Catholic Mission. You can get welfare. You won't see any Eskimo going at it white-fanged at whale or caribou meat. Na, na. They eat Kraft, bologna, and hot dogs plastered with H.P. sauce like everybody else. Which leads to further progress : every year there's a Dental Health Week. (p. 130,131)

Implantées dans une région des plus sauvages de l'est du Canada, les habitations de Fort Chimo sont regroupées autour d'un centre urbain très artificiel (billiards, Bingo). La précarité des logements («shacks») vient souligner l'aspect éphémère de l'agglomération où tout est fait pour répondre aux besoins de l'homme moderne, du cinéma au dentiste. La laideur du paysage se voit renforcée par la description de la situation du peuple Eskimo qui n'a d'égale que celle des Indiens dont la mention est récurrente tout au long du texte. Dénaturé par la civilisation à outrance, l'homme de souche se trouve lourdement affaibli et dépendant du système qu'on lui impose. Avec une pointe d'ironie, White critique la notion de Progrès. L'homme moderne, dans sa course frénétique au développement de l'activité industrielle, s'est coupé de la nature et a négligé son espace vital : "As you walk in the streets of Schefferville today... you look on with disgust at all the hurry-scurrying » (p. 105). La ville, (espace strié) est une surface fermée, répartie "suivant des intervalles déterminés, d'après des coupures assignées » (Mille Plateaux, p. 600). L'individu n'y est pas libre mais contrôlé, enchaîné et ne peut par conséquent que subir la vie. Il est loin d'avoir une grande santé et de posséder un gai savoir qui, libérateur d'énergies, le mettrait dans une position d'acteur, de créateur. Le mot « disgust » souligne la réaction physique provoquée par la vue du monde urbain, que l'auteur associe par conséquent au nauséeux et à l'indigeste.

Melville spoke of «ontological heroes ». There's a love of the world in them - as well as a disgust with what humanity makes of it - an immense, an encyclopaedic love, and an expense of their own persons that can amount to an ecstatic annihilation. (p. 56)

La réaction de rejet entraînée par la civilisation contraste avec « l'amour du monde ». Si les héros ontologiques de Melville ont un regard critique sur l'humanité, ce ne sont pas pour autant des figures du nihilisme mais, et nous reviendrons en aval sur ce point, de ce que White appelle le surnihilisme. Là où l'amour du monde et de la connaissance permette à l'être de s'émanciper, le monde urbain joue un rôle inverse en l'atrophiant. Le corps humain est affaibli par l'étroitesse de l'espace qui lui est alloué. La ville est présentée comme un espace exigu où l'énergie créatrice de l'homme et ses chances de 
s'affranchir s'amenuisent. Dès lors Kenneth White préconise une sortie du milieu urbain ainsi qu'un dégagement du carcan idéologique qui l'accompagne. Le lieu est non seulement un espace (physique) de vie, mais également un espace pour l'esprit. Il va de soi qu'une simple excursion en dehors des murs de la ville ne permettrait pas de recouvrer l'essence de l'être, le travail de désencombrement et de sape doit aussi se faire au sein même de la pensée.

Le «bloc » culturel attaqué par White trouve ses fondements dans la philosophie de Platon. Le rationalisme, le dualisme et le monde des idées (immatériel par nature) relayent au second plan le monde sensible. Il existe chez Platon une vérité supérieure et un savoir authentique qui gouvernent la pensée et ne permettent pas, selon la formule de Protagoras, à «l'homme d'être la mesure de toutes choses ${ }^{3}$ ». Les grandes lignes de ce ferment idéologique ont été reprises par le christianisme. La croyance en Dieu, le péché originel, les tables de la loi ou encore les arrière-mondes ne cessent de hanter les esprits et enchaînent l'individu à une idéologie rigide. Le platonisme et le christianisme sont les organes de la pensée occidentale. Selon White, c'est en structurant celle-ci de manière trop rigide qu'ils l'ont dévitalisée et qu'ils lui ont fait perdre le sens de la création. Le prosélytisme européen, qui commença par les explorations de Jacques Cartier aux $\mathrm{xv}^{e}$ et $\mathrm{xVI}^{\mathrm{e}}$ siècles, a profondément changé les habitudes des peuples animistes qui habitent le nord-est du Canada. Dans The Blue Road, White fait un constat d'échec, il mentionne et critique à plusieurs reprises la Bible ( « le gros livre ») qui depuis plus de deux mille ans forge les esprits du monde chrétien. L'accent est mis sur les divergences existentielles qui séparent la culture animiste (indienne et eskimo) de celle des chrétiens.

I wonder if we'll ever get rid of all this evangelical toponomy. I can't say what this lake's Indian name was, but I'm willing to bet it was beautiful, and precise too. Something like Blue Water Lake, or Summer Lake Storm, or Many Tree Lake. Named by people who knew it, who were in touch with its physical reality. But Lac SaintJean, I ask you... That's civilization, whatever be the book or the code. Nothing to do with beautifully felt reality. Which is why Flaubert could say civilization is a conspiracy against poetry. (p. 41, 42)

11 En nommant, l'homme connote les qualités qui appartiennent au lieu ou au sujet concerné. Il y a systématiquement un encodage; en revanche cet encodage peut être de différentes natures. White oppose deux démarches, celle des chrétiens et celle des indigènes. Les premiers ne se soucient guère de l'adéquation entre le lieu et le toponyme, les seconds recherchent la transparence. "Blue Water Lake», "Summer Lake Storm ", "Many Tree Lake » ne renvoient pas à un saint, mais à la singularité du lieu. Couleurs, éléments, saisons, météo et végétation sont à l'honneur dans la terminologie indienne imaginée par White. Pour que le lieu soit un espace où l'esprit puisse se développer librement, il est nécessaire qu'il ne soit pas pré-défini. Le nom Saint-Jean strie l'espace, il en fait un pur produit de l'espèce humaine, là où le poète (Flaubert, White...) aimerait voir disparaître ce surplus de culture. En striant l'espace, en le christianisant, l'homme apprivoise et dénature le lieu. Si White s'attaque à la codification qui découle des religions du Livre ${ }^{4}$ c'est pour tenter, dans un mouvement de dégagement, de retrouver l'essence du lieu. Lieu (non-lieu) qui, nous le voyons, s'annonce plus comme un espace pour l'esprit qu'une propriété du clergé ou de l'État.

Le travail de sape et de dégagement cède sa place à la phase de « reconstruction ». D'un espace strié le sujet se tourne vers un espace lisse, dans lequel il va tenter de se régénérer. 
If a man caught up in Western exile wants to find his Orient, he has to go through a passage North... Maybe the idea is to go as far as possible - to the end of yourself till you get into a territory where time turns into space, where things appear in all their nakedness and the wind blows anonymously. (p. 12)

Le voyage physique est prolégomènes à un changement radical. Le passage par le Nord est symbolique, le voyageur emprunte un sentier en marge afin de se déconditionner. Ce passage le mène au non-lieu, cet espace pour l'esprit. Si le lieu est purement géographique, le non-lieu est lui à cheval sur le géographique et l'ontologique : «They say in the Zen texts : when you get to the top of the mountain, keep climbing. Which is to say: when you get to the end of the road, keep going" (p. 96). Les concepts de Deleuze et de Guattari semblent ici à propos: le chemin et la route font partie des "espaces striés" alors que les territoires, qui se situent au-delà, dans le vide, constituent «l'espace lisse ». Les deux auteurs parlent d'ailleurs de «l'espace lisse du Zen » (Mille Plateaux, p. 468). La notion de vide, qui recoupe celle de l'espace lisse, est au cœur de la pensée orientale. Là où la philosophie occidentale associe la vacuité au néant, la pensée orientale y voit un souffle vital. C'est le souffle de l'origine, qui donne naissance à toute chose et qui garantit un renouvellement permanent. C'est en restant proche de cette source que la sensation de vie s'intensifie. Cette vision du monde ne se focalise pas sur la construction d'un système de pensée, il n'y pas de but à atteindre mais une jouissance à augmenter. White, après avoir mis l'accent sur la déconstruction, ne cherche pas tant à construire, qu'à "rester près de l'existence et de la pensée fragmentaire " (Une apocalypse tranquille, p. 107) garantes d'une sensation de vie plus forte. Cette sensation, synonyme d'intensité et de flux, s'accroît au sein d'un espace lisse et diminue dans un espace strié. Dès lors le sentier que cherche à emprunter le voyageur se tient en dehors de tout code. White parle à ce titre de «bird path» dans un poème qui se trouve dans l'antépénultième chapitre du récit : « moving in deeper/ever deeper/into a world/that is neither old nor new... on the bird path/feeling it out " (p. 129). Comme l'oiseau qui se meut en toute liberté le « je » se déplace dans un espace qui n'est pas borné et dont il jouit pleinement. Le sujet est maître de ses mouvements, il suit, trace ou définit sa propre voix avec la plus grande liberté. Le déplacement, qui se fait à tâtons («feeling it out»), souligne la retenue qu'il y a dans le geste ainsi que la dimension corporelle et sensuelle (tâter : de tangere " toucher »). Le " bird path », c'est le sentier qui n'est pas défini, le sentier atopique par excellence, symbole d'une nouvelle façon de se déplacer, d'habiter et d'être au monde.

Le logos suppose une langue fondée sur « ce qui est clair pour les sens comme pour l'âme » (Whitman), capable d'exprimer le monde blanc et d'y conduire l'homme afin que, au lieu de tourner en rond dans un cercle vicieux en tant qu'animal rationale (en proie à une logique qui ne peut que l'enfoncer davantage dans sa prison), il puisse devenir animal poeticum: non une victime de son environnement dégradé, mais un habitant $d u$ monde où il respire. (mes italiques), (La figure du dehors, p. 159-160).

Comment habiter poétiquement la terre ? Là où l'animal rationale serenferme sur-lui, l'animal poeticum devient une "figure du dehors ». Plusieurs protagonistes du récit incarnent cet animal poeticum. C'est le cas du glouton et illusionniste Carcajou, personnage du folklore indien et à qui est consacré le chapitre seize de The Blue Road:

Carcajou... is always running around the world, always jumping about from place to

place... Whatever happens, always making it, always merry and bright, always full

of tricks... He deinflates the

culture. He makes you get your feet back on the ground. (p. 111) 
Carcajou évolue en dehors de tout code. Il ne se fixe en aucun lieu, s'en prend à la culture qu'il «dégonfle » et reste fidèle à la terre. Il ne cherche pas à élaborer une pensée systémique et rigide mais à rester en mouvement. La vision du monde de Carcajou est proche de celle que Nietzsche envisageait pour son surhomme : « que tout corps devienne danseur et que tout esprit devienne oiseau " (Zarathroustra, p. 278). Il est important de souligner la place du corps dans la philosophie nietzschéenne et la " grande santé » qu'il considérait être au centre de ses préoccupations. Les errances folles de Carcajou, qui reflètent celles de l'auteur, démontrent non seulement l'engouement que suscitent le déplacement et la rencontre d'autrui, mais sont également le signe d'une santé hors-norme :

J'affirme une santé, la possibilité d'une santé, liée à une capacité de se mouvoir, désencombré, dans l'espace atopique, capacité qui ne peut venir que de l'acquisition d'une autre pensée, d'une autre manière d'être. (L $a$ Figure du dehors, p. 146)

Si White tire à boulets rouges sur la logique rationaliste c'est qu'il lui reproche de ne pas permettre à l'individu de se réaliser pleinement et de le rendre victime du monde qu'il ne sait habiter. À travers son œuvre il s'efforce de retrouver l'énergie créatrice qui gît à la source de l'être et ce via un retour à la terre et à l'être archaïque. "La rêverie nous apprend que l'essence de l'être, c'est le bien être enraciné dans l'être archaïque » (Gaston Bachelard, Poétique de la rêverie) Cette phrase de Gaston Bachelard, que White aime citer, exprime le désir de recouvrer l'essence de l'être. La mise en scène d'une figure légendaire comme celle de Carcajou montre à l'homme l'attitude à adopter. Il évolue dans «la voie du dehors, en dehors de toute forme limitée » (Scènes d'un monde flottant, p.110) et il est emblématique de ce que White appelle le surnihiliste. Le surnihiliste est l'être réalisé, celui qui a atteint l'absolu de lui-même et jouit de la vie.

Mes cinq étapes : Christianisme (sauvage et christique, pas jésuistique). Humanisme (révolté, pas conventionnel et classiciste). Égoïsme (transcendantal, pas borné). Nihilisme (négativiste et joyeux, pas désespéré). Surnihilsme (atopique, pas défini, en cours). (Scènes d'un monde flottant, p. 110)

La synthèse du développement personnel de l'auteur montre son attachement à l'idée de désencombrement. La dernière étape, qui se veut atopique et indéterminée, se traduit par une joie de vivre intense. Dans le poème qui clôt le récit (Labrador or the Waking Dream), le protagoniste, qui n'est d'ailleurs plus le narrateur ${ }^{5}$, témoigne de la jouissance qu'il éprouve au contact du lieu :

I lived and moved

as I had never done before

became a little more than human even

knew a larger identity

the tracks of the caribou in the snow

the flying of wild geese

the red autumn of the maple tree

bitten by frost

all these became more real to me

more really me

than my very name

I found myself saying things like

"at one with the spirit of the land"

but there was no "spirit", none

that outworn language

and this was a new world

and my mind was, almost, a new mind... (p. 139,140) 
Au terme de son périple le sujet se renouvelle profondément, prend conscience de son être véritable et, pour reprendre les termes de Nietzsche, semble « être devenu ce qu'il est » (cf. Ecce Homo). Le changement de narrateur est à cet égard significatif. Le moi de l'auteur est absent de la fin du récit, son importance devient secondaire. Il est par conséquent "un événement parmi d'autres " (Le Réel et son Double, p.111), non plus cloisonné dans son moi intime mais réalisé et ouvert sur l'extérieur. C'est en se réalisant qu'il accède au réel (« all these became more real to me »), ou à l'inverse en accédant au réel qu'il se réalise. Le "réel» joue un rôle primordial, il constitue la dernière étape du processus, comme l'est le Labrador pour Kenneth White (« Labrador is where I come full circle »). Cette région sauvage est à la frontière du lieu et du nonlieu, elle est le site où se déploie le réel. Mais le réel est, à l'instar du non-lieu, difficile à concevoir et donc à définir (cf. "Surnihilisme (atopique, pas défini, en cours) »). Le réel s'oppose au fantasme, au fictif, à l'imaginaire ("there was no "spirit" »). Le réel est simple (pas double) et s'appréhende mieux via la "matière, éternellement riche et vivante » qu'à travers des "événements insignifiants" (Le Réel et son Double, p. 110). Le réel, c'est ce qui reste une fois que le travail d'épure a été mené à son terme. Ceci explique la place des espaces vierges (déserts, steppes ou côtes découpées) qui vont être le site où le réel s'établit.

Pas de soi qui ne soit que soi, pas d'ici qui ne soit qu'ici, pas de maintenant qui ne soit que maintenant: telle est l'exigence du double... Ce refus de l'unique n'est d'ailleurs qu'une des formes les plus générales du refus de la vie. C'est pourquoi l'élimination $\mathrm{du}$ double annonce au contraire le retour en force du réel et se confond avec la joie d'un matin tout neuf... (Le Réel et son Double, p. 97)

Le poème Labrador or the Waking Dream commence par les vers suivants: "Another dawn/out from Greenland». En franchissant un seuil, symbole "d'un matin tout neuf », le protagoniste semble pénétrer le réel. Au contact de celui-ci le «je » prends conscience de son être véritable, («more really me/than my very name»), il n'est plus un autre mais lui-même, il a éliminé son double. Le double du « je » était constitué du religieux, du mythologique, du métaphysique, du culturel au sens le plus large ${ }^{6}$. C'est en se débarrassant progressivement de cette charge qu'il accède au réel. L'identité s'émancipe, le sujet se meut librement et vit de manière plus intense. L'homme fusionne avec la terre d'où il tire son bien-être (" the tracks of the caribou in the snow/ the flying of the wild geese/the red autumn of the maple tree bitten by frost/all these became more real to me »). Il ne possède plus la nature mais en fait partie. Un nouveau moi et un nouveau monde émergent de ce rapport rafraîchi avec la terre. L'intensification du sentiment de vie recherchée à travers le processus de désencombrement est éprouvée, une nouvelle vie commence. Le caractère non-fini, non-codifié propre à l'espace atopique, ou espace lisse, donne une plus grande liberté aux flux d'énergie, aux forces créatrices qui le constituent et le régénèrent perpétuellement. Mais comment faire en sorte que ces flux et ces forces ne cessent, comment conserver cette dynamique, cette grande santé ? La réponse est donnée par White dans l'avant-dernier chapitre de The Blue Road: « in the space won at the end of the personal journey, identities and times are mingled. Beyond ourselves, then, let's listen to the poem » (p. 134). Le poème, et Labrador en est un exemple magistral, permet de pénétrer l'esprit de la vie, de "pénétrer dans le monde de cette "vraie vie" dont Rimbaud déplorait l'absence ${ }^{7}$ » et à son écoute se déploie une énergie capable de maintenir l'être dans son état de jouissance. 

et la question du lieu dans la pensée de Kenneth White. La singularité et la puissance du langage poétique ont pour effet de résister au processus d'enfermement. L'énergie qu'il dégage plonge l'écrivain et le lecteur dans un espace qui ne se clôture pas, un espace qui reste indéfini, en mouvement. "L'infini, en nous, n'est-ce pas la langue, en ses combinatoires et ses virtualités, tel qu'à travers elle nous nous tenons face au fini et $\mathrm{y}$ descellons de l'infini: un en deçà, un au-delà, un avers, une opacité et un inconnaissable» (Le Poète perplexe, p. 359). Maupoix met en corrélation l'infini, le nonborné ou encore l'espace lisse avec le langage : à langage dynamique (poétique) espace ouvert. Le poème est dès lors un objet dont le rôle est de véhiculer ces flux d'énergie. À ce stade le langage poétique a moins une valeur communicative qu'affective. L'affect, cet état affectif élémentaire, " est la décharge rapide de l'émotion ", il « renvoie à des vitesses et à des compositions de vitesses entre elles » Mille Plateaux, p. 497, 498). La fin du poème Labrador éclaire à ce sujet la démarche de White : «I have cut these runes on a rock/to be my testament/perhaps no one will read them... beside the scratchings of the ice/open to wind and weather ». L'écriture runique est précisément liée à la question de l'affect : « dans sa première période, le runique n'a qu'une faible valeur de communication, et une fonction publique très réduite. Son caractère secret fait qu'on l'a souvent interprété comme une écriture magique. Il s'agit plutôt d'une sémiotique affective... elle formerait un texte ornemental plutôt que scripturaire " (Mille Plateaux, p. 500). L'intérêt que porte White aux runes est lié au fait qu'elles ne codifient et ne fixent pas le sens de manière définitive. Cette sémiotique affective se traduit par le jeu de forces, «de vitesses et de compositions de vitesses entre elles». L'écriture défie le sens, elle en atteint les limites et le langage a des pouvoirs autres que ceux qui lui sont communément attribués. Tout se passe comme si le langage n'avait plus son départ «au petit face-à-face Homme/Homme, mais au grand face-à-face Univers/Homme ${ }^{8}$ » et que sa force, qui est à son comble dans le langage poétique, était en mesure de garder l'être dans l'ouvert, dans un lieu non défini (atopique), clef de voûte permettant de lutter contre la clôture.

\section{BIBLIOGRAPHIE}

\section{Textes de Kenneth White}

La Figure du dehors, Paris, Grasset, 1982,

Scènes d'un monde flottant, Poitiers, Grasset, 1983.

The Blue Road, New York, George Braziller, Inc, 1984.

Une apocalypse tranquille, Paris, Grasset, 1985.

Travels in the Drifting Dawn, London, Penguin, 1989.

Pilgrim of the Void, Edinburgh, Mainstream Publishing, 1992.

Une stratégie paradoxale, Talence, Presses Universitaires de Bordeaux, 1998.

Open World, The Collected Poems 1960-2000, Edinburgh, Polygon, 2003.

\section{Bibliographie sélective}


CHAUCHE C., Langue et Monde. Grammaire géopoétique du paysage contemporain, Paris, L'Harmattan, 2004.

DELEUZE G., Guattari, F., Mille Plateaux, Lonrai, Les Éditions de Minuit, 2006.

MAUPOIX, Le Poète perplexe, Corti, 2002.

NIETZSCHE F., Zarathoustra, La Flèche (Sartre), Classiques de Poche, 1983.

ROSSET C., Le Réel et son Double, Saint-Amand, Folio, « Essais », 2002.

\section{NOTES}

1. Fourier Charles (1772-1837): il critiqua la société industrielle bourgeoise. L'organisation sociétaire qu'il prône a pour centre la phalange (phalanstère). Cette organisation doit avoir pour résultat l'harmonie universelle. Ce projet utopique ne put se réaliser, mais le fouriérisme eut des adeptes (Le Petit Robert).

2. L'Anti-@Edipe et Mille Plateaux sont mentionnés à plusieurs reprises dans l'œuvre de Kenneth White. Signalons également que White est cité dans Mille Plateaux (p. 470), dans le chapitre Traité de nomadologie : la machine de guerre. Il est évident que le thème de la création qui est au cœur de la pensée deleuzienne fait écho au travail de White.

3. Célèbre phrase attribuée au philosophe grec Protagoras ; l'ouvrage qui la contenait est perdu, mais Platon, dans son dialogue Théétète, la rappelle par la voix du mathématicien Théodore.

4. Il faut distinguer ici le «livre » en tant que bible ou livre saint qui codifie la pensée, du livre d'auteur qui vient bousculer les codes et ouvrir, en la nourrissant, la pensée du lecteur.

5. Kenneth White, Collected Poems, p. 622, 623: «The protagonist of this poem is one of those Scandinavian sea-travellers like Leif Ericson or Thorfinn Karlsefni who were active around the tenth century, and who touched on American shores. "

6. Dans la seconde section du poème ce point est rendu explicite : «I was no longer Christian/nor yet had I gone back to Thor... I tried to learn/the language of that silence/more difficult than the Latin/I learned in Bergen/Or the Irish in Dublin », p. 138, p. 139.

7. White, K., La Figure du dehors, p. 163. NB : Les affinités de White avec Rimbaud sont maintes fois mentionnées par l'auteur. Deleuze et Guattari rapprochent également les deux poètes dans Mille Plateaux, en traitant la question de la tribu : « une tribu dans le désert au lieu d'un sujet universel sous l'horizon de l'Être englobant. », p. 470.

8. Chauche C., Langue et Monde, Grammaire géopoétique du paysage contemporain. NB : C. Chauche cite le linguiste Gustave Guillaume.

\section{AUTEUR}

\section{CHRISTOPHE RONCATO}

Université Stendhal-Grenoble 3 